
\title{
25 Research Sourere \\ Using multimodal MRI to investigate alterations in brain structure and function in rats with type 2 diabetes.
}

\section{Christopher M Lawson}

Center for Translational Neurolmaging, Northeastern University, Boston, Massachusetts

\section{Kilian FG Rentrup}

Center for Translational Neurolmaging, Northeastern University, Boston, Massachusetts

\section{Xuezhu Cai}

Center for Translational Neurolmaging, Northeastern University, Boston, Massachusetts

\section{Praveen P Kulkarni}

Center for Translational Neurolmaging, Northeastern University, Boston, Massachusetts

Craig Ferris ( $\nabla$ c.ferris@northeastern.edu )

Northeastern University https://orcid.org/0000-0001-9744-5214

\section{Research}

Keywords: magnetic resonance imaging, small vessel disease, BBZDR/Wor rat, diffusion weighted imaging, voxel-based morphometry, resting state BOLD functional imaging

Posted Date: March 13th, 2020

DOI: https://doi.org/10.21203/rs.3.rs-17165/v1

License: (c) (1) This work is licensed under a Creative Commons Attribution 4.0 International License. Read Full License 


\section{Abstract}

Objectives This is an exploratory study using multimodal magnetic resonance imaging (MRI) to interrogate the brain of rats with type 2 diabetes (T2DM) as compared to controls. It was assumed there would be changes in brain structure and function that reflected the human disorder, thus providing a model system by which to follow disease progression with non-invasive MRI.

Methods The transgenic BBZDR/Wor rat, an animal model of T2MD, and age-matched controls were studied for changes in brain structure using voxel-based morphometry, alteration in white and gray matter microarchitecture using diffusion weighted imaging with indices of anisotropy, and functional coupling using resting state BOLD functional connectivity. Images from each modality were registered to, and analyzed, using a 3D MRI rat atlas providing site-specific data on over 168 different brain areas.

Results There was an overall reduction in brain volume focused primarily on somatosensory cortex, cerebellum and white matter tracts. The putative changes in white and gray matter microarchitecture were pervasive affecting much of the brain and not localized to any region. There was a general increase in connectivity in T2DM rats as compared to controls. The cerebellum presented with strong functional coupling to pons and brainstem in T2DM rats but negative connectivity to hippocampus.

Conclusion Are the neuroradiological measures collected in BBBKZ/Wor rats using multimodal imaging methods common to the clinic, similar to those reported in T2DM patents? In comparison to the clinical findings, the data would suggest the BBBKZ/Wor rat is not an appropriate imaging model for T2DM.

\section{Background}

Diabetes is a serious metabolic disorder estimated to affect 30 million people in the US as of 2016, with prevalence expected to reach more than 54.9 million Americans by 2030 [1, 2]. Diabetes is broken down into two main categories, type 1 diabetes (T1DM) and type 2 diabetes (T2DM). Destruction of pancreatic beta cells resulting in insulin deficiency is the hallmark of T1DM while T2DM is typically the result of a combination of peripheral insulin resistance and dysfunctional insulin secretion by pancreatic beta cells [3]. T2DM is much more common in the United States with $90-95 \%$ of all diabetes cases being such [1]. The pathology of T2MD is systemic, affecting much of the body and most functions. The brain is not spared as there are severe effects on cognition and behavior with disease progression and aging [4]. Studies with magnetic resonance imaging (MRI) report abnormalities in cerebral macrostructure and microstructure such as cortical atrophy [5], regional reductions in brain volume [6], structural deformities in cerebral gray matter [7], increased white matter lesions [8, 9] and changes in blood brain barrier permeability [10]. Indeed, there is a large body of literature using multimodal MRI e.g. voxel based morphometry (VBM), diffusion weighted imaging (DWI) and resting state BOLD functional MRI (rsFC), to interrogate brain structure and function in T2DM patients to better understand disease progression and prognosis for cognitive decline (for review see [11]). 
Animal models using magnetic resonance spectroscopy (MRS) have provided insights into the cellular and molecular mechanisms contributing to the metabolic disorders underpinning the cerebral neuropathy of T2DM [12-15]. For example, a recent study by Duarte and colleagues used ${ }^{13} \mathrm{CMRS}$ at ultrahigh field strength (14.1 T) to follow in vivo disruption in energy metabolism and impairment in the glutamateglutamine cycle between neurons and astrocytes in the Goto-Kakizaki (GK) rat model of T2DM [15]. While these types of animal studies are critical to understanding the neurodegeneration associated with T2DM, they cannot be performed on humans using clinical scanners. Indeed, there is a paucity of MRI studies in animal models of T2DM using imaging modalities commonly performed in the clinic. We know of only two such studies, one looking at ischemic vascular damage and axonal density following stroke in the high-fat diet, streptozotocin treated Wistar rat (HFD/STZ) [16], and a second in the TALLYHO/JngJ (TH) mouse correlating white matter connectivity using DWI with compulsive behavior [17]. Hence, the major advantage of non-invasive animal imaging - to follow disease progression with the same imaging modalities used in the clinic - has not been exploited. To address this short coming, we performed an exploratory study using VBM, DWI and rsFC to interrogate the brain of the obese Bio-Breeding Zucker diabetic (BBZDR/Wor) rat, a model of T2DM [18]. Our findings are discussed in the context of their clinical relevance and whether this animal model and the imaging modalities used would have translational value in a larger prospective study following evolution of cerebral neuropathy in T2DM.

\section{Methods}

\section{Animal Model}

Male Bio-Breeding Zucker diabetic rats (BBZDR/Wor rats) $(n=8)$ as well as age-matched non-diabetic BBDR littermates $(n=8)$, were obtained from Biomere in Worcester, MA for imaging. The BBZDR/Wor rat is an inbred rat strain of T2DM and is emerging as a model to study the many complications that encompass T2DM in humans. In BBZDR/Wor animals, the recessive Iddm2 gene responsible for lymphopenia and spontaneous autoimmunity is removed while the Lepr $\mathrm{fa}$ ( $\mathrm{fa} 1$ ) mutation is retained. Obese male BBZDR/Wor rat spontaneously develops type 2 diabetes at approximately 10 weeks of age ( 100\%) when fed standard rat chow [18-21]. The BBZDR/Wor diabetic rat displays the clinical symptoms typically associated with T2DM including dyslipidemia, hyperglycemia, insulin resistance, hypertension, and decreased levels of the beta cell-specific glucose transporter type-2 (GLUT-2) [18, 19, 22].

Rats were maintained on a $12 \mathrm{~h}: 12 \mathrm{~h}$ light:dark cycle with a lights on at 07:00 h, allowed access to food and water ad libitum and were treated with IP injections of saline at indications of weight loss. The average age of the animals at time of imaging was about 4 months. All animal experiments were conducted in accordance with the Northeastern University Division of Laboratory Animal Medicine and Institutional Animal Care and Use Committee.

\section{Neuroimaging}


Imaging was conducted using a Bruker Biospec 7.0T/20-cm USR horizontal magnet (Bruker, Billerica, MA, USA) and a $20-\mathrm{G} / \mathrm{cm}$ magnetic field gradient insert (ID = $12 \mathrm{~cm}$ ) capable of a $120-\mu$ s rise time. Radio frequency signals were sent and received with a quadrature volume coil built into the animal restrainer (Animal Imaging Research, Holden, Massachusetts). All rats were restrained using a custom restraint kit and imaged under $1-2 \%$ isoflurane while keeping a respiratory rate of $40-50 / \mathrm{min}$.

\section{Voxel-Based Morphometry}

Images were acquired using RARE sequence with TR/TE $=3310 / 36 \mathrm{~ms}$; matrix size $256 \times 256 \times 40$, field of view $=30 \times 30 \mathrm{~mm}$, spatial resolution, $0.117 \times 0.117 \times 0.7 \mathrm{~mm}$. A 3D MRI Rat Brain Atlas (@) 2012 Ekam Solutions LLC, Boston, MA) was used to calculate brain volumes, and registered the standard structural rat template image onto high resolution T2-weighted images for each subject using a non-linear registration method implemented by Unix based software package Deformable Registration via Attribute Matching and Mutual-Saliency Weighting (DRAMMS;

https://www.cbica.upenn.edu/sbia/software/dramms/index.html). The atlas (image size $256 \times 256 \times 63$ ) $(\mathrm{H} \times \mathrm{W} \times \mathrm{D})$ was then warped from the standard space into the subject image space (image size $256 \times$ $256 \times 40$ ) using the deformation obtained from the above step using nearest-neighbor interpolation method. In the volumetric analysis, each brain region was therefore segmented, and the volume values were extracted for all 171 ROIs, calculated by multiplying unit volume of voxel in $\mathrm{mm}^{3}$ by the number of voxels using an in-house MATLAB script. To account for different brain sizes, all ROI volumes were normalized by dividing each subject's ROI volume by their total brain volume

\section{Diffusion Weighted Imaging - Quantitative Anisotropy}

DWI was acquired with a spin-echo echo-planar-imaging (EPI) pulse sequence having the following parameters: TR/TE $=500 / 20$ msec, eight EPI segments, and 10 non-collinear gradient directions with a single b-value shell at $1000 \mathrm{~s} / \mathrm{mm} 2$ and one image with a B-value of $0 \mathrm{~s} / \mathrm{mm} 2$ (referred to as B 0 ). Geometrical parameters were: 48 coronal slices, each $0.313 \mathrm{~mm}$ thick (brain volume) and with in-plane resolution of $0.313 \times 0.313 \mathrm{~mm} 2$ (matrix size $96 \times 96$; FOV $30 \mathrm{~mm}^{3}$ ). The imaging protocol was repeated two times for signal averaging. Each DWI acquisition took $35 \mathrm{~min}$ and the entire MRI protocol lasted about 70 min. Image analysis included DWI analysis of the DW-3D-EPI images to produce the maps of fractional anisotropy (FA) and apparent diffusion coefficient (ADC). DWI analysis was completed with MATLAB and

MedINRIA (1.9.0; http://www-sop.inria.fr/asclepios/software/MedINRIA/index.php) software. Because sporadic excessive breathing during DWI acquisition can lead to significant image motion artifacts that are apparent only in the slices sampled when motion occurred, each image (for each slice and each gradient direction) was screened, prior to DWI analysis. If found, acquisition points with motion artifacts were eliminated from analyses.

For statistical comparisons between rats, each brain volume was registered to the 3D rat atlas allowing voxel- and region-based statistics. All image transformations and statistical analyses were carried out 
using the in-house MIVA software (http://ccni.wpi.edu/). For each rat, the B 0 image was co-registered with the B 0 template (using a 6-parameter rigid-body transformation). The co-registration parameters were then applied on the DWI indexed maps for the different indices of anisotropy. Normalization was performed on the maps since they provided the most detailed visualization of brain structures and allow for more accurate normalization. The normalization parameters were then applied to all DWI indexed maps that were then smoothed with a 0.3-mm Gaussian kernel. To ensure that FA and ADC values were not affected significantly by the pre-processing steps, the 'nearest neighbor' option was used following registration and normalization.

Statistical differences in measures of DWI between experimental groups were determined using a nonparametric Mann-Whitney U Test (alpha set at 5\%). The formula below was used to account for false discovery from multiple comparisons.

$$
P_{(i)} \leq \frac{i}{V} \quad \frac{q}{c(V)}
$$

$\mathrm{P}(\mathrm{i})$ is the $\mathrm{p}$ value based on the $t$ test analysis. Each of 171 ROls (i) within the brain containing $(\mathrm{V}) \mathrm{ROIs}$ was ranked in order of its probability value (see Table 1 ). The false-positive filter value q was set to 0.2 and the predetermined $c(V)$ was set to unity 11 . The corrected probability is noted on each table. 
Table 1

Apparent Diffusion Coefficient

\begin{tabular}{|c|c|c|c|c|c|c|c|}
\hline \multicolumn{8}{|l|}{ Apparent Diffusion Coefficient } \\
\hline \multirow[b]{2}{*}{ Brain Area } & \multicolumn{3}{|c|}{ Control } & \multicolumn{4}{|c|}{ BBDRZ/Wo } \\
\hline & Ave & SD & & Ave & SD & P-val & $\Omega s q$ \\
\hline basal amygdaloid n. & 2.13 & 0.14 & $>$ & 1.87 & 0.07 & 0.002 & 0.722 \\
\hline lateral amygdaloid $\mathrm{n}$. & 2.20 & 0.14 & $>$ & 1.83 & 0.12 & 0.002 & 0.722 \\
\hline pontine reticular $\mathrm{n}$. caudal & 2.54 & 0.29 & $>$ & 2.05 & 0.19 & 0.002 & 0.722 \\
\hline parvicellular reticular $\mathrm{n}$. & 2.62 & 0.25 & $>$ & 1.83 & 0.39 & 0.002 & 0.720 \\
\hline caudal piriform ctx & 2.18 & 0.12 & $>$ & 1.61 & 0.13 & 0.002 & 0.720 \\
\hline gigantocellularis reticular $\mathrm{n}$. & 2.79 & 0.28 & $>$ & 2.03 & 0.42 & 0.002 & 0.719 \\
\hline retrosplenial rostral ctx & 2.55 & 0.22 & $>$ & 2.09 & 0.15 & 0.002 & 0.719 \\
\hline ventral subiculum & 2.42 & 0.29 & $>$ & 1.95 & 0.13 & 0.002 & 0.719 \\
\hline cortical amygdaloid n. & 2.42 & 0.22 & $>$ & 1.84 & 0.22 & 0.002 & 0.688 \\
\hline frontal association ctx & 2.59 & 0.15 & $>$ & 1.78 & 0.28 & 0.003 & 0.655 \\
\hline habenula $\mathrm{n}$. & 3.03 & 0.21 & $>$ & 2.37 & 0.30 & 0.003 & 0.653 \\
\hline root of trigeminal nerve & 3.12 & 0.26 & $>$ & 2.25 & 0.48 & 0.003 & 0.651 \\
\hline anterior lobe pituitary & 2.97 & 0.49 & $>$ & 1.60 & 0.72 & 0.003 & 0.651 \\
\hline CA3 hippocampus ventral & 2.29 & 0.18 & $>$ & 1.94 & 0.11 & 0.003 & 0.651 \\
\hline crus 1 of ansiform lobule & 2.19 & 0.34 & $>$ & 1.48 & 0.28 & 0.003 & 0.651 \\
\hline entorhinal ctx & 2.68 & 0.26 & $>$ & 1.97 & 0.20 & 0.003 & 0.651 \\
\hline glomerular layer & 2.66 & 0.12 & $>$ & 1.87 & 0.44 & 0.003 & 0.651 \\
\hline paraflocculus cerebellum & 2.29 & 0.18 & $>$ & 1.86 & 0.22 & 0.003 & 0.651 \\
\hline solitary tract $\mathrm{n}$. & 2.91 & 0.45 & $>$ & 1.97 & 0.36 & 0.003 & 0.651 \\
\hline superior colliculus & 2.57 & 0.23 & $>$ & 2.15 & 0.12 & 0.004 & 0.591 \\
\hline dorsomedial tegmental area & 2.57 & 0.29 & $>$ & 2.15 & 0.13 & 0.004 & 0.589 \\
\hline medial amygdaloid $\mathrm{n}$. & 2.84 & 0.21 & $>$ & 2.41 & 0.18 & 0.004 & 0.588 \\
\hline reticular $\mathrm{n}$. & 2.06 & 0.08 & $>$ & 1.87 & 0.11 & 0.005 & 0.558 \\
\hline CA1 hippocampus ventral & 2.14 & 0.21 & $>$ & 1.77 & 0.19 & 0.006 & 0.530 \\
\hline
\end{tabular}




\begin{tabular}{|llllllll|}
\hline \multicolumn{2}{|l}{ Apparent Diffusion Coefficient } \\
\hline 5th cerebellar lobule & 2.85 & 0.29 & $>$ & 2.16 & 0.30 & 0.006 & 0.529 \\
\hline inferior colliculus & 2.87 & 0.32 & $>$ & 2.39 & 0.18 & 0.006 & 0.529 \\
\hline visual 1 ctx & 2.30 & 0.31 & $>$ & 1.92 & 0.16 & 0.006 & 0.529 \\
\hline central amygdaloid n. & 2.42 & 0.23 & $>$ & 1.99 & 0.17 & 0.006 & 0.527 \\
\hline dentate gyrus ventral & 2.67 & 0.35 & $>$ & 2.13 & 0.16 & 0.006 & 0.527 \\
\hline vestibular n. & 2.86 & 0.38 & $>$ & 2.21 & 0.25 & 0.006 & 0.527 \\
\hline medial dorsal thalamic n. & 2.18 & 0.15 & $>$ & 1.90 & 0.18 & 0.007 & 0.501 \\
\hline
\end{tabular}

\section{Resting state functional connectivity}

Scans were collected using a spin-echo triple-shot EPI sequence (imaging parameters: matrix size $=96 \times$ $96 \times 20(\mathrm{H} \times \mathrm{W} \times \mathrm{D}), \mathrm{TR} / \mathrm{TE}=1000 / 15 \mathrm{msec}$, voxel size $=0.312 \times 0.312 \times 1.2 \mathrm{~mm}$, slice thickness $=1.2 \mathrm{~mm}$, with 200 repetitions, time of acquisition $10 \mathrm{~min}$. There are numerous studies detailing the benefits of multi-shot EPI in BOLD imaging [23-27]. We avoided using single shot EPI because of its severe geometrical distortion at high field strengths $(\geq 7 T)$ and loss of effective spatial resolution as the readout period increases $[24,28,29]$. There is also the possibility of signal loss in single shot EPI due to accumulated magnetic susceptibility or field inhomogeneity [27].

Preprocessing in this study was accomplished by combining Analysis of Functional Neurolmages (AFNI_17.1.12, http://afni.nimh.nih.gov/afni/), FMRIB Software library (FSL, v5.0.9, http://fsl.fmrib.ox.ac.uk/fsl/), Deformable Registration via Attribute Matching and Mutual-Saliency Weighting (DRAMMS 1.4.1,https://www.cbica.upenn.edu/sbia/software/dramms/index.html) and MATLAB (Mathworks,Natick, MA). Brain tissue masks for resting-state functional images were manually drawn using 3DSlicer (https://www.slicer.org/) and applied for skull-stripping. Motion outliers (i.e., data corrupted by extensive motion) were detected in the dataset and the corresponding time points were recorded so that they could be regressed out in a later step. Functional data were assessed for the presence of motion spikes. Any large motion spikes were identified and removed from the time-course signals. This filtering step was followed by slice timing correction from interleaved slice acquisition order. Head motion correction (six motion parameters) was carried out using the first volume as a reference image. Normalization was completed by registering functional data to the 3D MRI Rat Brain Atlas (C) 2012 Ekam Solutions LLC, Boston, MA) using affine registration through DRAMMS. The 3D MRI Rat Brain Atlas containing 171 annotated brain regions was used for segmentation. Data are reported in 166 brain areas, as five regions in the brain atlas were excluded from analysis due to the large size of three brains. These brains fell slightly outside our imaging field of view and thus we did not get any signal from the extreme caudal tip of the cerebellum. Whole brains that contain all regions of interest are needed for analyses so rather than excluding the animals, we removed the brain sites across all animals. After quality assurance, band-pass filtering $(0.01 \mathrm{~Hz} \sim 0.1 \mathrm{~Hz})$ was performed to reduce low-frequency drift 
effects and high-frequency physiological noise for each subject. The resulting images were further detrended and spatially smoothed (full width at half maximum $=0.8 \mathrm{~mm}$ ). Finally, regressors comprised of motion outliers, the six motion parameters, the mean white matter, and cerebrospinal fluid time series were fed into general linear models for nuisance regression to remove unwanted effects.

The region-to-region functional connectivity method was performed in this study to measure the correlations in spontaneous BOLD fluctuations. A network is comprised of nodes and edges; nodes being the brain region of interest $(\mathrm{ROI})$ and edges being the connections between regions. Data are reported in 166 brain areas, as five regions in the 3D MRI Rat Brain Atlas were excluded from analysis due to the large size of three brains that fell slightly outside then field of view excluding signal from the most caudal tip of the cerebellum. Voxel time series data were averaged in each node based on the residual images using the nuisance regression procedure. Pearson's correlation coefficients across all pairs of nodes (14535 pairs) were computed for each subject among all three groups to assess the interregional temporal correlations. The r-values (ranging from - 1 to 1 ) were $z$-transformed using the Fisher's $Z$ transform to improve normality. $166 \times 166$ symmetric connectivity matrices were constructed with each entry representing the strength of edge. Group-level analysis was performed to look at the functional connectivity in the experimental groups. The resulting Z-score matrices from one-group t-tests were clustered using the K-nearest neighbors clustering method to identify how nodes cluster together and form resting state networks. A Z-score threshold of $|Z|=2.3$ was applied to remove spurious or weak node connections for visualization purposes.

\section{Behavioral Tests}

The novel object recognition (NOR) task was attempted to assess episodic learning and memory [30, 31]. The apparatus consisted of a black cube-shaped Plexiglass box (L: 60.9, W: 69.2, H: $70.5 \mathrm{~cm}$ ) with no lid, indirectly illuminated with two $40 \mathrm{~W}$ incandescent bulbs. Animals were placed in the empty box ( $15 \mathrm{~min})$ for acclimation on day 1 . On day 2 , for the familiar phase $(5 \mathrm{~min})$, animals were placed in the box with two identical objects arranged in diagonal corners, $5 \mathrm{~cm}$ from each wall. After a 90 min rest period in their home cage, animals were placed back in the box for the novel phase $(3 \mathrm{~min})$ with one of the familiar objects and a novel object.

The cold plate assay was attempted to test for cold allodynia and was taken from Brenner and Golden (2012) [32] and modified for rats. Prior to testing, rats were acclimated to standing on a $1 / 4$ inch glass plate table, confined to an area of $38 \mathrm{~cm} \times 14 \mathrm{~cm}$ by a transparent plastic container. Beneath the glass table a mirror was positioned to permit a clear view of the rat's hind paws for the precise placement of the cold probe. A hollow probe with a tip dimension of $2 \mathrm{~mm}$ was filled with compressed, crushed dry ice. The center of the plantar surface of the hindpaw was targeted for stimulation through the floor of the glass platform. The cold probe was tested on each hindpaw with an interval of $7 \mathrm{~min}$ between each paw. Both hindpaws of each rat were tested three times with an interval of 15 min between trials. The latency to move the hindpaw away from the cold probe was timed in seconds. The maximum time allowed for withdrawal was $90 \mathrm{sec}$. Withdrawal latencies were averaged between the right and left hindpaw and did not differ significantly between paws within either the vehicle or paclitaxel treatments. Consequently, the 
right and left hindpaw withdrawal times were averaged for each animal, and a single mean determination from each animal contributed to the groups means and standard deviations for each treatment. These data were statistically compared with a Student's t-Test. Experimenters collecting the data were blinded to the treatment condition.

However, due the obesity of the diabetic rats, their mobility was extremely limited which prevented any accurate or meaningful recordings from either of the tests. Additionally, due to the rapid decline of the diabetic rats' health, subsequent behavioral tests that did not require mobility were not possible.

\section{Results}

\section{Voxel-Based Morphometry}

Shown in Fig. 1 is a table comparing the average brain volumes that were significantly different between control and BBZDR/Wor rats. The brain areas are ranked in order of their significance and are truncated from a larger list of 171 areas (see Supplementary Table S1). Note, in all cases the BBZDR/Wor brain volumes are smaller than controls. While not statistically significant, this is also true for a majority of brain areas, 136/171, as shown in Table S1. Shown to the left of Fig. 1 is a 3D reconstruction summarizing the brains areas listed in the table. Several areas of the somatosensory cortex (upper lip, hindlimb, forelimb, barrel field, entorhinal, temporal, and retrosplenial cortices) showed a reduced volume as did the white matter tracts, basal ganglia (striatum, substantia nigra, septum), anterior cerebellum (2nd, 3rd, 5th lobules) and olfactory bulbs (granular and external plexiform layers).

\section{Diffusion Weighted Imaging}

The significant differences in between control and BBZDR/Wor rats for ADC and FA are presented in Tables 1 and 2, respectively. The brain areas in each table are ranked in order of their significance. In the case of ADC, 90/171 brain areas were significantly different and for FA there were 111/171 significant brain areas. Only those brain areas with an effect size greater than 0.5 are listed. The areas in each case spread out to cover much the brain. BBZDR/Wor rats showed greater FA values and lower ADC values as compared to control. The full tables of brain areas are provided in Supplementary Tables S2 and S3. 
Table 2

Fractional Anisotropy

\begin{tabular}{|c|c|c|c|c|c|c|c|}
\hline \multicolumn{8}{|l|}{ Fractional Anisotropy } \\
\hline \multirow[b]{2}{*}{ Brain Area } & \multicolumn{3}{|c|}{ Control } & \multicolumn{4}{|c|}{ BBDRZ/Wo } \\
\hline & Ave & SD & & Ave & SD & P-val & $\Omega s q$ \\
\hline superior colliculus & 0.29 & 0.02 & $<$ & 0.39 & 0.06 & 0.002 & 0.745 \\
\hline auditory ctx & 0.26 & 0.02 & $<$ & 0.41 & 0.05 & 0.002 & 0.740 \\
\hline inferior colliculus & 0.30 & 0.02 & $<$ & 0.40 & 0.04 & 0.002 & 0.738 \\
\hline dentate gyrus dorsal & 0.28 & 0.02 & $<$ & 0.38 & 0.05 & 0.002 & 0.736 \\
\hline insular ctx & 0.30 & 0.02 & $<$ & 0.42 & 0.05 & 0.002 & 0.736 \\
\hline ventral lateral striatum & 0.28 & 0.02 & $<$ & 0.39 & 0.05 & 0.002 & 0.732 \\
\hline perirhinal ctx & 0.33 & 0.03 & $<$ & 0.47 & 0.04 & 0.002 & 0.730 \\
\hline ventral posteriolateral thalamic $n$. & 0.42 & 0.03 & $<$ & 0.52 & 0.03 & 0.002 & 0.730 \\
\hline reticular n. midbrain & 0.34 & 0.02 & $<$ & 0.46 & 0.08 & 0.002 & 0.728 \\
\hline rostral piriform ctx & 0.32 & 0.03 & $<$ & 0.43 & 0.04 & 0.002 & 0.726 \\
\hline primary somatosensory ctx jaw & 0.29 & 0.03 & $<$ & 0.41 & 0.06 & 0.002 & 0.726 \\
\hline subthalamic $n$. & 0.44 & 0.02 & $<$ & 0.53 & 0.03 & 0.002 & 0.726 \\
\hline basal amygdaloid $n$. & 0.32 & 0.05 & $<$ & 0.42 & 0.03 & 0.002 & 0.724 \\
\hline secondary motor ctx & 0.32 & 0.05 & $<$ & 0.44 & 0.04 & 0.002 & 0.724 \\
\hline posterior thalamic $\mathrm{n}$. & 0.30 & 0.02 & $<$ & 0.42 & 0.06 & 0.002 & 0.724 \\
\hline ventral posteriolmedial thalamic $n$. & 0.34 & 0.02 & $<$ & 0.46 & 0.07 & 0.002 & 0.724 \\
\hline accumbens core & 0.33 & 0.03 & $<$ & 0.44 & 0.04 & 0.002 & 0.722 \\
\hline anterior olfactory $n$. & 0.36 & 0.03 & $<$ & 0.47 & 0.05 & 0.002 & 0.722 \\
\hline claustrum & 0.32 & 0.02 & $<$ & 0.45 & 0.06 & 0.002 & 0.722 \\
\hline dorsal lateral striatum & 0.32 & 0.03 & $<$ & 0.42 & 0.05 & 0.002 & 0.722 \\
\hline endopiriform n. & 0.35 & 0.03 & $<$ & 0.46 & 0.03 & 0.002 & 0.722 \\
\hline lateral preoptic area & 0.31 & 0.02 & $<$ & 0.40 & 0.05 & 0.002 & 0.722 \\
\hline medial orbital ctx & 0.28 & 0.04 & $<$ & 0.45 & 0.11 & 0.002 & 0.722 \\
\hline secondary somaotsensory ctx & 0.25 & 0.02 & $<$ & 0.39 & 0.07 & 0.002 & 0.722 \\
\hline
\end{tabular}




\section{Fractional Anisotropy}

\begin{tabular}{|c|c|c|c|c|c|c|c|}
\hline ventral orbital ctx & 0.27 & 0.02 & $<$ & 0.44 & 0.07 & 0.002 & 0.722 \\
\hline CA1 hippocampus ventral & 0.28 & 0.06 & $<$ & 0.47 & 0.05 & 0.002 & 0.720 \\
\hline lateral orbital ctx & 0.28 & 0.02 & $<$ & 0.44 & 0.07 & 0.002 & 0.720 \\
\hline granular cell layer & 0.36 & 0.04 & $<$ & 0.51 & 0.04 & 0.002 & 0.719 \\
\hline dorsal medial striatum & 0.33 & 0.04 & $<$ & 0.44 & 0.06 & 0.002 & 0.695 \\
\hline parietal ctx & 0.32 & 0.03 & $<$ & 0.39 & 0.06 & 0.002 & 0.692 \\
\hline 3rd cerebellar lobule & 0.31 & 0.04 & $<$ & 0.44 & 0.05 & 0.002 & 0.690 \\
\hline parafascicular thalamic $n$. & 0.29 & 0.02 & $<$ & 0.39 & 0.08 & 0.002 & 0.690 \\
\hline primary somatosensory hindlimb & 0.29 & 0.03 & $<$ & 0.39 & 0.04 & 0.002 & 0.690 \\
\hline ventral subiculum & 0.33 & 0.04 & $<$ & 0.46 & 0.05 & 0.002 & 0.690 \\
\hline magnocellular preoptic $n$. & 0.34 & 0.05 & $<$ & 0.46 & 0.07 & 0.002 & 0.688 \\
\hline temporal ctx & 0.30 & 0.04 & $<$ & 0.41 & 0.05 & 0.002 & 0.688 \\
\hline prelimbic ctx & 0.31 & 0.04 & $<$ & 0.46 & 0.09 & 0.002 & 0.686 \\
\hline lateral geniculate & 0.31 & 0.03 & $<$ & 0.44 & 0.05 & 0.003 & 0.663 \\
\hline dentate gyrus ventral & 0.29 & 0.04 & $<$ & 0.46 & 0.06 & 0.003 & 0.662 \\
\hline anterior amygdaloid n. & 0.30 & 0.04 & $<$ & 0.41 & 0.04 & 0.003 & 0.660 \\
\hline lemniscal n. & 0.36 & 0.02 & $<$ & 0.44 & 0.07 & 0.003 & 0.660 \\
\hline 4th cerebellar lobule & 0.33 & 0.03 & $<$ & 0.43 & 0.05 & 0.003 & 0.655 \\
\hline entorhinal ctx & 0.33 & 0.03 & $<$ & 0.43 & 0.04 & 0.003 & 0.655 \\
\hline ventromedial thalamic $n$. & 0.33 & 0.04 & $<$ & 0.46 & 0.06 & 0.003 & 0.655 \\
\hline olfactory tubercles & 0.34 & 0.03 & $<$ & 0.47 & 0.06 & 0.003 & 0.653 \\
\hline medial preoptic area & 0.27 & 0.03 & $<$ & 0.38 & 0.07 & 0.003 & 0.627 \\
\hline reuniens $\mathrm{n}$. & 0.28 & 0.03 & $<$ & 0.38 & 0.06 & 0.003 & 0.627 \\
\hline primary somatosensory ctx upper lip & 0.26 & 0.03 & $<$ & 0.37 & 0.07 & 0.003 & 0.624 \\
\hline tenia tecta ctx & 0.28 & 0.03 & $<$ & 0.42 & 0.08 & 0.003 & 0.624 \\
\hline medial geniculate & 0.34 & 0.03 & $<$ & 0.47 & 0.09 & 0.003 & 0.622 \\
\hline Ventricle & 0.38 & 0.03 & $<$ & 0.45 & 0.04 & 0.004 & 0.600 \\
\hline primary somatosensory ctx trunk & 0.30 & 0.04 & $<$ & 0.38 & 0.06 & 0.004 & 0.597 \\
\hline
\end{tabular}




\begin{tabular}{|lllllll|}
\hline Fractional Anisotropy & & & & & & \\
\hline accumbens shell & 0.34 & 0.03 & $<0.44$ & 0.05 & 0.004 & 0.594 \\
\hline lateral posterior thalamic $\mathrm{n}$. & 0.28 & 0.04 & $<0.41$ & 0.06 & 0.004 & 0.594 \\
\hline infralimbic ctx & 0.30 & 0.04 & $<0.43$ & 0.06 & 0.004 & 0.592 \\
\hline CA2 & 0.28 & 0.04 & $<0.40$ & 0.07 & 0.004 & 0.591 \\
\hline primary somatosensory barrel field & 0.27 & 0.04 & $<0.39$ & 0.07 & 0.004 & 0.591 \\
\hline primary somatosensory shoulder & 0.27 & 0.04 & $<0.39$ & 0.06 & 0.004 & 0.591 \\
\hline trapezoid body & 0.38 & 0.06 & $<0.49$ & 0.04 & 0.004 & 0.591 \\
\hline 5th cerebellar lobule & 0.35 & 0.04 & $<0.43$ & 0.05 & 0.005 & 0.567 \\
\hline arcuate $\mathrm{n}$. & 0.31 & 0.05 & $<0.44$ & 0.09 & 0.005 & 0.563 \\
\hline 2nd cerebellar lobule & 0.35 & 0.05 & $<0.48$ & 0.08 & 0.005 & 0.561 \\
\hline CA3 hippocampus ventral & 0.32 & 0.07 & $<0.46$ & 0.04 & 0.005 & 0.561 \\
\hline external plexiform layer & 0.41 & 0.04 & $<0.50$ & 0.04 & 0.006 & 0.533 \\
\hline periaqueductal gray thalamus & 0.31 & $0.02<0.43$ & $<0.09$ & 0.006 & 0.533 \\
\hline ectorhinal ctx & 0.34 & 0.04 & $<0.42$ & 0.06 & 0.006 & 0.531 \\
\hline ventral medial striatum & 0.33 & 0.05 & $<0.42$ & 0.04 & 0.006 & 0.530 \\
\hline lateral amygdaloid $\mathrm{n}$. & 0.32 & 0.05 & $<0.44$ & 0.06 & 0.006 & 0.527 \\
\hline raphe linear & 0.37 & 0.04 & $<0.47$ & 0.07 & 0.007 & 0.505 \\
\hline zona incerta & 0.40 & 0.04 & $<0.54$ & 0.09 & 0.007 & 0.503 \\
\hline 8th cerebellar lobule & 0.34 & 0.05 & $<0.46$ & 0.07 & 0.007 & 0.501 \\
\hline ventrolateral thalamic $\mathrm{n}$. & 0.31 & 0.05 & $<0.40$ & 0.05 & 0.007 & 0.501 \\
\hline
\end{tabular}

Resting State Functional Connectivity

Figure 2 shows a correlation matrix comparing 166 brain areas for rsFC between the controls and BBZDR/Wor rats. Each colored red/orange pixel represents 1 of 166 brain areas that has a significant positive correlation with other brain areas. Pixels in shades of blue have a significant negative, or anticorrelation with other brain regions. The brain areas with significant correlations appear as clusters because they are contiguous in their neuroanatomy and function. The diagonal line separates the control and BBZDR/Wor groups. Each pixel on one side of the line has a mirror image pixel on the other side. The delineated areas serve to focus attention on similarities and differences in connectivity. Area A shows intra-thalamic connections and favors greater coupling for BBZDR/Wor rats. B is the posterior thalamus and $\mathrm{C}$ the midbrain. $\mathrm{D}$ shows connections between the habenula/tectal/parafascicular thalamus and the 
dorsal hippocampus with clear hypoconnectivity in BBZDR/Wor as compared to control. E highlights the area of the prefrontal cortex, and $\mathrm{F}$ the cerebellum/pons. G shows a broad area of connections between the cerebellum/pons and the trigeminal brainstem reticular activating system again favoring higher coupling in BBZDR/Wor rats as compared to controls. Area I is the brainstem reticular activating system and deep cerebellar nuclei. Of particular interest is the anti-correlation or uncoupling in area $\mathrm{H}$ (blue pixels) between the hippocampus and the posterior cerebellum. A table is provided showing the positive (yellow) and negative (blue) connections with the dorsal hippocampus (CA1, CA3 and dentate) highlighted in red. The $Z$ score to the right of each brain area is the average score to the three areas of the dorsal hippocampus. For example, the lateral geniculate has significant connections to CA1, CA3 and dorsal dentate (denoted by the 3 shown in parentheses) the average of which is a $Z$ score of 4 . In contrast, the crus 2 of the cerebellum only has significant negative connections to two of the three hippocampal areas (denoted by two in parentheses) with an average $Z$ score of negative 2.9. This relationship between hippocampus, thalamus and cerebellum/brainstem is highlighted in 3D reconstructions to the right. Shown is the positive coupling between the dorsal hippocampus and multiple thalamic nuclei and the uncoupling or negative correlation to dorsal striatum, posterior cerebellum and brainstem.

\section{Discussion}

There are several rat models that may have translational benefits when using MRI to study T2DM [33]. These include the GK rat [34], Zuker Diabetic Fatty (ZDF) rat [35], HFD/STZ rat [36] and BBBZK/Wor, to list a few. This exploratory study began the process of evaluating the BBBZK/Wor model of T2DM with multimodal imaging with the expectation of finding common neuroradiological measures of cerebral neuropathy as reported in the clinic. If so, the model would have the potential to provide valuable imaging data on disease progression from prediabetic to late stage diabetes and a non-invasive means of assessing the efficacy of therapeutic intervention on brain structure and function.

\section{Voxel-Based Morphometry}

A reduction in brain volume is a consistent finding across all imaging studies in 2TDB [11]. Cortical volumes show a decrease in gray $[6,37-40]$ and white matter $[6,40]$. The underlying cause of the global reduction in brain volumes is unknown but is thought to be the consequence of small vessel disease [41]. The general brain atrophy and reduction in gray matter is associated with diminished cognitive function $[37,40,42,43]$. Hippocampal volumes are smaller in T2DM versus age-matched controls $[40,44,45]$ and maybe a contributing factor to the cognitive decline. The BBBZK/Wor rat presented with several brain areas that were significantly smaller as compared to age-matched controls. These areas included the somatosensory, entorhinal and temporal cortices but not CA1, CA3, dentate, and subiculum, the primary components of the hippocampal complex.

\section{Diffusion weighted imaging}


DWI is an indirect way of assessing the integrity of white and gray matter microarchitecture. A recently published study by Moghaddam and colleagues [46] provides a comprehensive review of the literature on T2DM and DWI. The literature is very consistent reporting changes in microarchitecture in the areas of the frontal-temporal cortex, hippocampus, cerebellum, thalamus and all of the major white matter tracts. The cognitive decline in T2DM is strongly associated with alterations in DWI in white matter tracts [47-49]. With few exceptions, the general DWI profile is a decrease in FA and increase in ADC. This inverse relationship suggests a loss of microstructural integrity and network organization [46]. The BBBZK/Wor rat also presented with global and pervasive changes in measures of DWI. The FA and ADC values were inversely related with FA being greater than ADC a potential sign of global neuroinflammation and cytotoxic edema. The changes in DWI were extensive affecting much of the brain including the hippocampus, thalamus, amygdala, cerebellum and white matter tracts.

\section{Functional Connectivity}

A review by Macpherson and colleagues covers much of the literature on rsFC in T2DM [50]. There is general agreement across multiple studies that T2DM presents with a reduction in connectivity in the default mode network, interconnections between the prefrontal cortex, parietal cortex, and hippocampus. Thalamic coupling to cortical and cerebellar regions is also reduced [51]. The effect on global connectivity in this BBZDR/Wor diabetes model is contrary to that reported in the clinical literature. As shown in the connectivity matrix Fig 2 there is no clear reduction in connectivity when looking at the clusters highlighted. If anything, there are areas of the brain that show hyperconnectivity with diabetes. Intra-thalamic connections are enhanced (area A), as are thalamic connections to the dorsal hippocampus (area D) and cerebellar/pontine connections to the brainstem reticular activating system (area G). The increase in connectivity is not unprecedented and can occur between some brain areas in human T2DM [52], but that would appear to be the exception. The hyperconnectivity observed in BBBZK/Wor maybe a compensatory response to underlying pathology as reported in traumatic brain injury [53] or in young children with early type 1 diabetes [54].

\section{Limitations and Considerations}

As an exploratory study we recognize its many limitations. 1) There were no female rats, an issue of concern given data shows differences in diabetic pathology between females and males $[55,56] 2)$ There were no measures of cognitive function to correlation with the MRI data as is routine with clinical studies. As noted in the Methods, we were unable to collect behavioral data due to the severity of the obesity in the BBBKZ/Wor rat. 3) We did not image for white matter hyperintensities, lesions associated with microvascular insult. Again, this is routine in clinical studies and would have aided in our analysis of BBBKZ/Wor as a relevant imaging model for T2DM. 4) Our rsFC studies were done under light isoflurane anesthesia. These studies could have been done under awake conditions as we have done so in many other task-related BOLD imaging [57-59] or phMRI studies $[60,61]$ in rodents. However, "resting state" 
poses a dilemma in awake animal imaging no matter the level of acclimation prior to imaging [62]. Any physical restraint will most likely have some level of stress; hence, the rsFC data were collected under light anesthesia. Nonetheless, numerous studies comparing the anesthetized and conscious states show similar rsFC data $[63,64]$

\section{Limitations And Considerations}

As an exploratory study we recognize its many limitations. 1) There were no female rats, an issue of concern given data shows differences in diabetic pathology between females and males $[55,56] 2)$ There were no measures of cognitive function to correlation with the MRI data as is routine with clinical studies. As noted in the Methods, we were unable to collect behavioral data due to the severity of the obesity in the BBBKZ/Wor rat. 3) We did not image for white matter hyperintensities, lesions associated with microvascular insult. Again, this is routine in clinical studies and would have aided in our analysis of BBBKZ/Wor as a relevant imaging model for T2DM. 4) Our rsFC studies were done under light isoflurane anesthesia. These studies could have been done under awake conditions as we have done so in many other task-related BOLD imaging [57-59] or phMRI studies $[60,61]$ in rodents. However, "resting state" poses a dilemma in awake animal imaging no matter the level of acclimation prior to imaging [62]. Any physical restraint will most likely have some level of stress; hence, the rsFC data were collected under light anesthesia. Nonetheless, numerous studies comparing the anesthetized and conscious states show similar rsFC data $[63,64]$

\section{Conclusion}

These limitations aside, the original question stands - are the neuroradiological measures collected in BBBKZ/Wor rats using multimodoal imaging methods common to the clinic, similar to those reported in T2DM patents? While the VBM data in BBBKZ/Wor reflected the general findings in T2DM showing a decrease in specific brain areas and a global trend toward a reduction in volume, the hippocampus, a critical area linking changes with cognitive function to disease progression, was unaffected. The DWI changes in FA and ADC were global and pervasive with no specific areas that could be identified as potential biomarkers by which to follow the prediabetic to diabetic stages of cerebral neuropathy. The rsFC data were contrary to most findings in T2DM which report hypoconnectivity with disease progression. The hyperconnectivity in BBBKZ/Wor rat most likely reflects an effort to compensate for the pathology that is not seen in humans. From these observations, we would conclude the BBBKZ/Wor rat is not an appropriate imaging model for T2DM.

\section{Declarations}

\section{Ethics approval and consent to participate}

Not applicable 


\section{Consent for publication}

Yes

\section{Availability of data and material}

All data can be accessed through a link to Mandeley. DOI to follow

\section{Competing interests}

CFF has a financial interest in Animal Imaging Research, the company that makes the RF electronics and holders for animal imaging

\section{Funding}

None

\section{Authors' contributions}

All of the authors have contributed substantially to the manuscript.

Concept, drafting and interpretation - Ferris, Lawson, Rentrup

Execution and analysis - Cai, Kulkarni, Lawson, Rentrup

\section{Acknowledgements}

We thank Biomere (Worcester, Massachusetts, USA) for providing the BBZDR/Wor rats and their agematched non-diabetic BBDR littermates for these studies.

\section{References}

1. Prevention CfDCa: National Diabetes Statistics Report, 2017. (Atlanta GCfDCaP, US Department of Health and Human Services ed.; 2017.

2. Rowley WR, Bezold C, Arikan Y, Byrne E, Krohe S: Diabetes 2030: Insights from Yesterday, Today, and Future Trends. Popul Health Manag 2017, 20:6-12.

3. Ndisang JF, Vannacci A, Rastogi S: Insulin Resistance, Type 1 and Type 2 Diabetes, and Related Complications 2017. J Diabetes Res 2017, 2017:1478294.

4. Biessels GJ, van der Heide LP, Kamal A, Bleys RL, Gispen WH: Ageing and diabetes: implications for brain function. Eur J Pharmacol 2002, 441:1-14.

5. Schmidt R, Launer LJ, Nilsson LG, Pajak A, Sans S, Berger K, Breteler MM, de Ridder M, Dufouil C, Fuhrer $\mathrm{R}$, et al: Magnetic resonance imaging of the brain in diabetes: the Cardiovascular Determinants of Dementia (CASCADE) Study. Diabetes 2004, 53:687-692.

6. Last D, Alsop DC, Abduljalil AM, Marquis RP, de Bazelaire C, Hu K, Cavallerano J, Novak V: Global and regional effects of type 2 diabetes on brain tissue volumes and cerebral vasoreactivity. Diabetes Care 2007, 30:1193-1199. 
7. Zhang Y, Zhang X, Zhang J, Liu C, Yuan Q, Yin X, Wei L, Cui J, Tao R, Wei P, Wang J: Gray matter volume abnormalities in type 2 diabetes mellitus with and without mild cognitive impairment. Neurosci Lett 2014, 562:1-6.

8. Araki Y, Nomura M, Tanaka H, Yamamoto H, Yamamoto T, Tsukaguchi I, Nakamura H: MRI of the brain in diabetes mellitus. Neuroradiology 1994, 36:101-103.

9. Manschot SM, Brands AM, van der Grond J, Kessels RP, Algra A, Kappelle LJ, Biessels GJ, Utrecht Diabetic Encephalopathy Study G: Brain magnetic resonance imaging correlates of impaired cognition in patients with type 2 diabetes. Diabetes 2006, 55:1106-1113.

10. Starr JM, Wardlaw J, Ferguson K, MacLullich A, Deary IJ, Marshall I: Increased blood-brain barrier permeability in type II diabetes demonstrated by gadolinium magnetic resonance imaging. J Neurol Neurosurg Psychiatry 2003, 74:70-76.

11. Brundel M, Kappelle LJ, Biessels GJ: Brain imaging in type 2 diabetes. Eur Neuropsychopharmacol 2014, 24:1967-1981.

12. van der Graaf M, Janssen SW, van Asten JJ, Hermus AR, Sweep CG, Pikkemaat JA, Martens GJ, Heerschap A: Metabolic profile of the hippocampus of Zucker Diabetic Fatty rats assessed by in vivo $1 \mathrm{H}$ magnetic resonance spectroscopy. NMR Biomed 2004, 17:405-410.

13. Duarte JMN, Skoug C, Silva HB, Carvalho RA, Gruetter R, Cunha RA: Impact of Caffeine Consumption on Type 2 Diabetes-Induced Spatial Memory Impairment and Neurochemical Alterations in the Hippocampus. Front Neurosci 2018, 12:1015.

14. Soares AF, Nissen JD, Garcia-Serrano AM, Nussbaum SS, Waagepetersen HS, Duarte JMN: Glycogen metabolism is impaired in the brain of male type 2 diabetic Goto-Kakizaki rats. J Neurosci Res 2019, 97:1004-1017.

15. Girault FM, Sonnay S, Gruetter R, Duarte JMN: Alterations of Brain Energy Metabolism in Type 2 Diabetic Goto-Kakizaki Rats Measured In Vivo by (13)C Magnetic Resonance Spectroscopy. Neurotox Res 2019, 36:268-278.

16. Ding G, Yan T, Chen J, Chopp M, Li L, Li Q, Cui C, Ning R, Jiang Q: Persistent cerebrovascular damage after stroke in type two diabetic rats measured by magnetic resonance imaging. Stroke 2015, 46:507512.

17. van de Vondervoort I, Amiri H, Bruchhage MMK, Oomen CA, Rustogi N, Cooper JD, van Asten JJA, Heerschap A, Bahn S, Williams SCR, et al: Converging evidence points towards a role of insulin signaling in regulating compulsive behavior. Trans/ Psychiatry 2019, 9:225.

18. Tirabassi RS, Flanagan JF, Wu T, Kislauskis EH, Birckbichler PJ, Guberski DL: The BBZDR/Wor Rat Model for Investigating the Complications of Type 2 Diabetes Mellitus. ILAR Journa/2004, 45:292302.

19. Ellis EA, Grant MB, Murray FT, Wachowski MB, Guberski DL, Kubilis PS, Lutty GA: Increased NADH Oxidase Activity in the Retina of the BBZ/WOR Diabetic Rat. Free Radic Biol Med 1998, 24:111-120.

20. Ellis EA, Guberski DL, Hutson B, Grant MB: Time Course of NADH Oxidase, Inducible Nitric Oxide Synthase and Peroxynitrite in Diabetic Retinopathy in the BBZ/WOR Rat. Nitric Oxide 2002, 6:295- 
304.

21. Ellis EA, Guberski DL, Somogyi-Mann M, Grant MB: Increased H2O2, vascular endothelial growth factor and receptors in the retina of the BBZ/WOR diabetic rat. Free Radic Biol Med 2000, 28:91-101.

22. Sima A, Merry A, Hall D, Grant M, Murray F, Guberski DJE, Endocrinology C, Diabetes: The BBZ/WORDR-rat: A model for Type II diabetic neuropathy. 1997, 105:63-63.

23. Menon RS, Thomas CG, Gati JS: Investigation of BOLD contrast in fMRI using multi-shot EPI. NMR Biomed 1997, 10:179-182.

24. Hoogenraad FG, Pouwels PJ, Hofman MB, Rombouts SA, Lavini C, Leach MO, Haacke EM: Highresolution segmented EPI in a motor task fMRI study. Magn Reson Imaging 2000, 18:405-409.

25. Poser BA, Norris DG: Investigating the benefits of multi-echo EPI for fMRI at 7 T. Neuroimage 2009, 45:1162-1172.

26. Swisher JD, Sexton JA, Gatenby JC, Gore JC, Tong F: Multishot versus single-shot pulse sequences in very high field fMRI: a comparison using retinotopic mapping. PLoS One 2012, 7:e34626.

27. Kang D, Sung YW, Kang CK: Fast Imaging Technique for fMRI: Consecutive Multishot Echo Planar Imaging Accelerated with GRAPPA Technique. Biomed Res Int 2015, 2015:394213.

28. Farzaneh F, Riederer SJ, Pelc NJ: Analysis of T2 limitations and off-resonance effects on spatial resolution and artifacts in echo-planar imaging. Magn Reson Med 1990, 14:123-139.

29. Jesmanowicz A, Bandettini PA, Hyde JS: Single-shot half k-space high-resolution gradient-recalled EPI for fMRI at 3 Tesla. Magn Reson Med1998, 40:754-762.

30. Bevins RA, Besheer JJNp: Object recognition in rats and mice: a one-trial non-matching-to-sample learning task to study'recognition memory'. 2006, 1:1306.

31. Antunes M, Biala GJCp: The novel object recognition memory: neurobiology, test procedure, and its modifications. 2012, 13:93-110.

32. Brenner DS, Golden JP, Gereau RWt: A novel behavioral assay for measuring cold sensation in mice. PLoS One 2012, 7:e39765.

33. Al-Awar A, Kupai K, Veszelka M, Szucs G, Attieh Z, Murlasits Z, Torok S, Posa A, Varga C: Experimental Diabetes Mellitus in Different Animal Models. J Diabetes Res 2016, 2016:9051426.

34. Kuwabara WMT, Panveloski-Costa AC, Yokota CNF, Pereira JNB, Filho JM, Torres RP, Hirabara SM, Curi R, Alba-Loureiro TC: Comparison of Goto-Kakizaki rats and high fat diet-induced obese rats: Are they reliable models to study Type 2 Diabetes mellitus? PLoS One 2017, 12:e0189622.

35. Wang X, DuBois DC, Sukumaran S, Ayyar V, Jusko WJ, Almon RR: Variability in Zucker diabetic fatty rats: differences in disease progression in hyperglycemic and normoglycemic animals. Diabetes Metab Syndr Obes 2014, 7:531-541.

36. Srinivasan K, Viswanad B, Asrat L, Kaul CL, Ramarao P: Combination of high-fat diet-fed and lowdose streptozotocin-treated rat: a model for type 2 diabetes and pharmacological screening.

Pharmacol Res 2005, 52:313-320. 
37. Espeland MA, Bryan RN, Goveas JS, Robinson JG, Siddiqui MS, Liu S, Hogan PE, Casanova R, Coker $\mathrm{LH}$, Yaffe K, et al: Influence of type 2 diabetes on brain volumes and changes in brain volumes: results from the Women's Health Initiative Magnetic Resonance Imaging studies. Diabetes Care 2013, 36:90-97.

38. Jongen C, van der Grond J, Kappelle LJ, Biessels GJ, Viergever MA, Pluim JP, Utrecht Diabetic Encephalopathy Study G: Automated measurement of brain and white matter lesion volume in type 2 diabetes mellitus. Diabetologia 2007, 50:1509-1516.

39. Kumar A, Haroon E, Darwin C, Pham D, Ajilore O, Rodriguez G, Mintz J: Gray matter prefrontal changes in type 2 diabetes detected using MRI. J Magn Reson Imaging 2008, 27:14-19.

40. Moran C, Phan TG, Chen J, Blizzard L, Beare R, Venn A, Munch G, Wood AG, Forbes J, Greenaway TM, et al: Brain atrophy in type 2 diabetes: regional distribution and influence on cognition. Diabetes Care 2013, 36:4036-4042.

41. Kloppenborg RP, Nederkoorn PJ, Grool AM, Vincken KL, Mali WP, Vermeulen M, van der Graaf Y, Geerlings MI, Group SS: Cerebral small-vessel disease and progression of brain atrophy: the SMARTMR study. Neurology 2012, 79:2029-2036.

42. van Elderen SG, de Roos A, de Craen AJ, Westendorp RG, Blauw GJ, Jukema JW, Bollen EL, Middelkoop HA, van Buchem MA, van der Grond J: Progression of brain atrophy and cognitive decline in diabetes mellitus: a 3-year follow-up. Neurology 2010, 75:997-1002.

43. Wu G, Lin L, Zhang Q, Wu J: Brain gray matter changes in type 2 diabetes mellitus: A meta-analysis of whole-brain voxel-based morphometry study. J Diabetes Complications 2017, 31:1698-1703.

44. Gold SM, Dziobek I, Sweat V, Tirsi A, Rogers K, Bruehl H, Tsui W, Richardson S, Javier E, Convit A: Hippocampal damage and memory impairments as possible early brain complications of type 2 diabetes. Diabetologia 2007, 50:711-719.

45. Zhang YW, Zhang JQ, Liu C, Wei P, Zhang X, Yuan QY, Yin XT, Wei LQ, Cui JG, Wang J: Memory dysfunction in type 2 diabetes mellitus correlates with reduced hippocampal CA1 and subiculum volumes. Chin Med J (Engl) 2015, 128:465-471.

46. Sanjari Moghaddam H, Ghazi Sherbaf F, Aarabi MH: Brain microstructural abnormalities in type 2 diabetes mellitus: A systematic review of diffusion tensor imaging studies. Front Neuroendocrinol 2019, 55:100782.

47. Yau PL, Javier D, Tsui W, Sweat V, Bruehl H, Borod JC, Convit A: Emotional and neutral declarative memory impairments and associated white matter microstructural abnormalities in adults with type 2 diabetes. Psychiatry Res 2009, 174:223-230.

48. Hsu JL, Chen YL, Leu JG, Jaw FS, Lee CH, Tsai YF, Hsu CY, Bai CH, Leemans A: Microstructural white matter abnormalities in type 2 diabetes mellitus: a diffusion tensor imaging study. Neuroimage 2012, 59:1098-1105.

49. Reijmer YD, Brundel M, de Bresser J, Kappelle LJ, Leemans A, Biessels GJ, Utrecht Vascular Cognitive Impairment Study G: Microstructural white matter abnormalities and cognitive functioning in type 2 diabetes: a diffusion tensor imaging study. Diabetes Care 2013, 36:137-144. 
50. Macpherson H, Formica M, Harris E, Daly RM: Brain functional alterations in Type 2 Diabetes - A systematic review of fMRI studies. Front Neuroendocrinol 2017, 47:34-46.

51. Chen YC, Xia W, Qian C, Ding J, Ju S, Teng GJ: Thalamic resting-state functional connectivity: disruption in patients with type 2 diabetes. Metab Brain Dis 2015, 30:1227-1236.

52. Cui Y, Jiao Y, Chen HJ, Ding J, Luo B, Peng CY, Ju SH, Teng GJ: Aberrant functional connectivity of default-mode network in type 2 diabetes patients. Eur Radiol 2015, 25:3238-3246.

53. Kulkarni P, Morrison TR, Cai X, Iriah S, Simon N, Sabrick J, Neuroth L, Ferris CF: Neuroradiological Changes Following Single or Repetitive Mild TBI. Front Syst Neurosci 2019, 13:34.

54. Saggar M, Tsalikian E, Mauras N, Mazaika P, White NH, Weinzimer S, Buckingham B, Hershey T, Reiss AL, Diabetes Research in Children N: Compensatory Hyperconnectivity in Developing Brains of Young Children With Type 1 Diabetes. Diabetes 2017, 66:754-762.

55. Kautzky-Willer A, Harreiter J, Pacini G: Sex and Gender Differences in Risk, Pathophysiology and Complications of Type 2 Diabetes Mellitus. Endocr Rev 2016, 37:278-316.

56. Harreiter J, Kautzky-Willer A: Sex and Gender Differences in Prevention of Type 2 Diabetes. Front Endocrinol (Lausanne) 2018, 9:220.

57. Iriah SC, Trivedi M, Kenkel W, Grant SE, Moore K, Yee JR, Madularu D, Kulkarni P, Ferris CF: Oxycodone Exposure: A Magnetic Resonance Imaging Study in Response to Acute and Chronic Oxycodone Treatment in Rats. Neuroscience 2019, 398:88-101.

58. Yee JR, Kenkel WM, Kulkarni P, Moore K, Perkeybile AM, Toddes S, Amacker JA, Carter CS, Ferris CF: BOLD fMRI in awake prairie voles: A platform for translational social and affective neuroscience. Neuroimage 2016, 138:221-232.

59. Kenkel WM, Yee JR, Moore K, Madularu D, Kulkarni P, Gamber K, Nedelman M, Ferris CF: Functional magnetic resonance imaging in awake transgenic fragile $X$ rats: evidence of dysregulation in reward processing in the mesolimbic/habenular neural circuit. Trans/ Psychiatry 2016, 6:e763.

60. Ferris CF, Kulkarni P, Yee JR, Nedelman M, de Jong IEM: The Serotonin Receptor 6 Antagonist Idalopirdine and Acetylcholinesterase Inhibitor Donepezil Have Synergistic Effects on Brain Activity-A Functional MRI Study in the Awake Rat. Front Pharmacol 2017, 8:279.

61. Ferris CF, Yee JR, Kenkel WM, Dumais KM, Moore K, Veenema AH, Kulkarni P, Perkybile AM, Carter CS: Distinct BOLD Activation Profiles Following Central and Peripheral Oxytocin Administration in Awake Rats. Front Behav Neurosci 2015, 9:245.

62. Ferris CF, Smerkers B, Kulkarni P, Caffrey M, Afacan O, Toddes S, Stolberg T, Febo M: Functional magnetic resonance imaging in awake animals. Rev Neurosci 2011, 22:665-674.

63. Gorges M, Roselli F, Muller HP, Ludolph AC, Rasche V, Kassubek J: Functional Connectivity Mapping in the Animal Model: Principles and Applications of Resting-State fMRI. Front Neurol 2017, 8:200.

64. Jonckers E, Delgado y Palacios R, Shah D, Guglielmetti C, Verhoye M, Van der Linden A: Different anesthesia regimes modulate the functional connectivity outcome in mice. Magn Reson Med 2014, 72:1103-1112. 


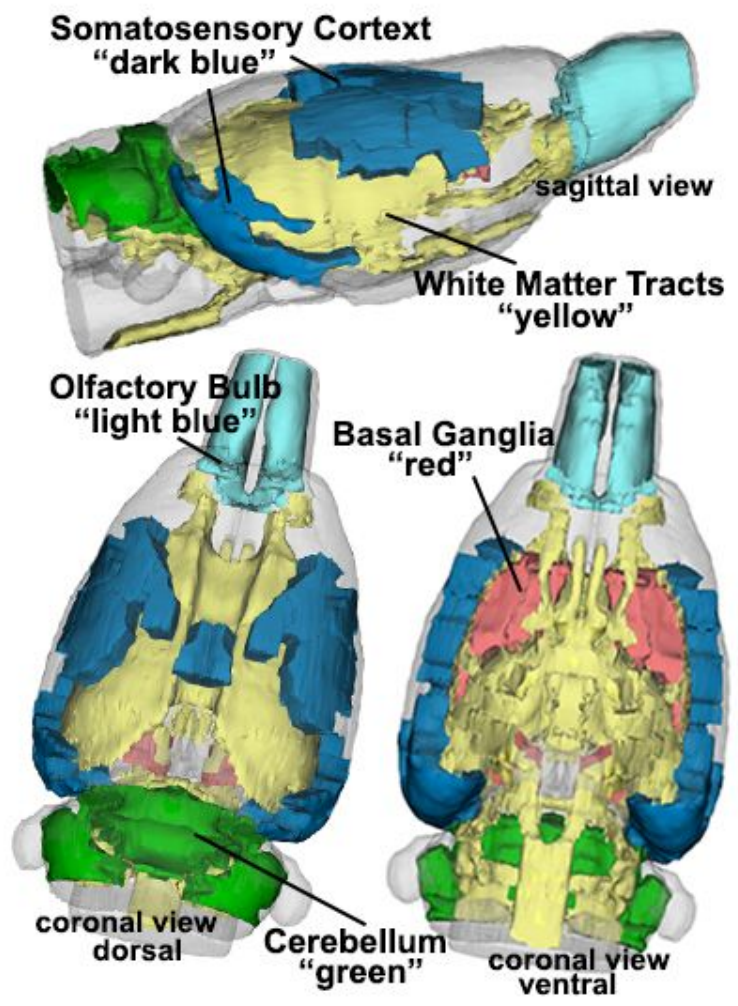

\begin{tabular}{|c|c|c|c|c|c|c|}
\hline \multicolumn{7}{|c|}{ Change in Brain Volume (mm3) } \\
\hline \multirow[b]{2}{*}{ Brain Area } & \multicolumn{2}{|c|}{ Control } & \multicolumn{3}{|c|}{ Diabetic } & \multirow[b]{2}{*}{ P-val } \\
\hline & Avg & SD & & Avg & SD & \\
\hline primary somatosensory ctx upper lip & 21.05 & 1.32 & $>$ & 18.80 & 1.58 & 0.008 \\
\hline 5th cerebellar lobule & 28.30 & 1.86 & $>$ & 25.48 & 1.92 & 0.010 \\
\hline substantia nigra compacta & 1.87 & 0.30 & $>$ & 1.47 & 0.23 & 0.010 \\
\hline 3rd cerebellar lobule & 19.33 & 1.81 & $>$ & 16.34 & 2.62 & 0.019 \\
\hline crus 1 of ansiform lobule & 34.02 & 2.81 & $>$ & 30.08 & 3.16 & 0.020 \\
\hline primary somatosensory ctx hindlimb & 11.23 & 0.57 & $>$ & 9.91 & 1.34 & 0.023 \\
\hline anterior lobe pituitary & 6.52 & 0.57 & $>$ & 5.56 & 0.94 & 0.027 \\
\hline dorsal medial striatum & 19.46 & 1.16 & $>$ & 17.59 & 1.84 & 0.029 \\
\hline external plexiform layer & 28.68 & 1.74 & $>$ & 26.54 & 1.80 & 0.029 \\
\hline pontine nuclei & 10.48 & 0.54 & $>$ & 9.38 & 1.20 & 0.033 \\
\hline dorsal lateral striatum & 29.36 & 2.11 & $>$ & 27.05 & 1.79 & 0.034 \\
\hline temporal ctx & 5.79 & 0.91 & $>$ & 4.96 & 0.43 & 0.036 \\
\hline primary somatosensory ctx forelimb & 20.53 & 1.45 & $>$ & 18.90 & 1.37 & 0.036 \\
\hline primary somatosensory ctx barrel field & 34.77 & 3.78 & $>$ & 30.82 & 2.98 & 0.036 \\
\hline retrosplenial rostral ctx & 8.99 & 0.31 & $>$ & 8.32 & 0.78 & 0.039 \\
\hline ventral lateral striatum & 25.12 & 1.73 & $>$ & 22.99 & 2.05 & 0.041 \\
\hline lateral septal nucleus & 16.69 & 1.70 & $>$ & 14.97 & 1.39 & 0.044 \\
\hline white matter & 82.53 & 5.29 & $>$ & 76.43 & 5.87 & 0.046 \\
\hline entorhinal ctx & 37.65 & 3.94 & $>$ & 33.18 & 4.23 & 0.046 \\
\hline 2nd cerebellar lobule & 15.53 & 1.73 & $>$ & 13.17 & 2.53 & 0.047 \\
\hline granular cell layer & 29.74 & 1.62 & $>$ & 27.74 & 2.07 & 0.049 \\
\hline
\end{tabular}

\section{Figure 1}

Voxel-based morphometry The table to the right list the brain areas that have significantly different volumes between experimental conditions. These brains areas are shown in the 3D reconstructions summarizing the differences.
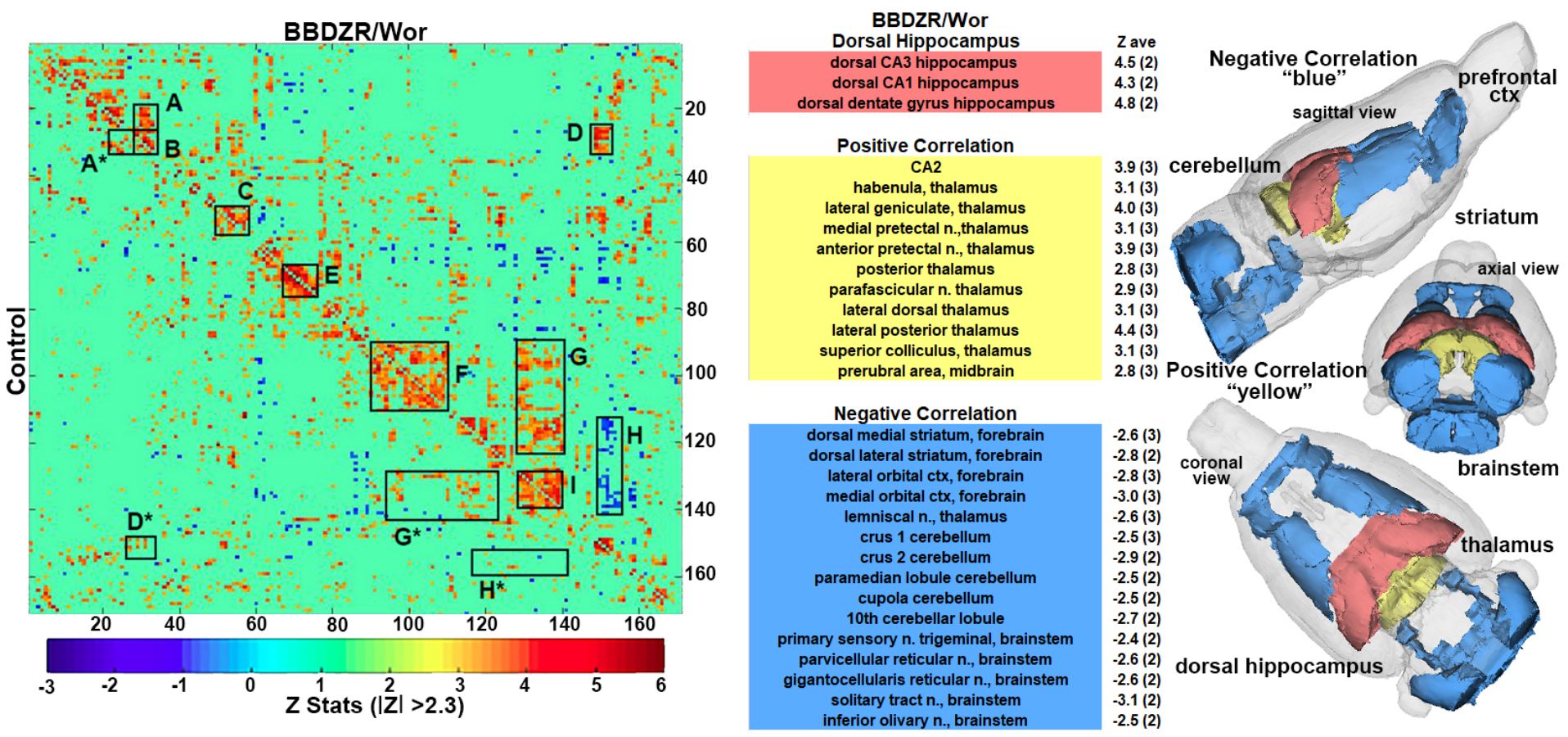


\section{Figure 2}

Resting Sate Functional Connectivity To the left is a correlation data matrix comparing control and BBZDR/Wor rats. To the right are tables of positively (yellow) and negatively (blue) coupled areas to the dorsal hippocampus (red) with 3D reconstruction summarizing the brain areas. A - intra-thalamic connections; B - posterior thalamus; C - midbrain, D connections between the habenula/tectal/parafascicular thalamus and the dorsal hippocampus; E- prefrontal cortex; F cerebellum/pons; $\mathrm{G}$ - connections between the cerebellum/pons and the trigeminal brainstem reticular activating system; $\mathrm{H}$ - hippocampus and the posterior cerebellum; I is the brainstem reticular activating system and deep cerebellar nuclei

\section{Supplementary Files}

This is a list of supplementary files associated with this preprint. Click to download.

- S2FullTable1ADC.xlsx

- S3FullTable1ADC.xIsx

- SupplementaryTable1BrainVolumes.xlsx 